\title{
Applications to Development of PET/SPECT System by Use of Geant4
}

\author{
Yoshiyuki Hirano \\ Department of Bio-medical Imaging \\ National Cerebral and Cardiovascular Center Research Institute
}

Japan

\section{Introduction}

In this chapter, two applications of Monte Carlo method in medicine are described. The first application is development of a Single Photon Emission Computed Tomography (SPECT), in which the intrinsic spatial and energy resolutions were estimated by simulating scintillation lights. The adequacy of the simulation was verified by comparisons of experimental results from a high-resolution SPECT. In addition, we proposed a method related to improving the spatial resolution relative to the conventional method, (the Anger method). The usefulness of the method and the performance were estimated by the simulation. The second application is an evaluation of a quantification technique for Positron Emission Tomography (PET). In cardiac PET using the isotope O-15, scatter events originating from outside the field of view (FOV), especially from the liver, influence the quantification of myocardial blood flow. We evaluated this influence by simulating trajectories of gamma rays. In section 1, we briefly describe Monte Carlo simulation tools in medicine and principle of PET/SPECT imaging. In sections 2 and 3, respectively, we describe applications to SPECT and PET.

\subsection{Monte Carlo simulation in medicine}

Monte Carlo simulation is widely used in development of medical equipments. In development of PET and SPECT, Monte Carlo simulation is a useful tool for understanding the trajectories of gamma rays emitted from radiopharmaceuticals. We employed a Monte Carlo simulation library, Geant4 (Allison J et al., 2006) written in C++. Geant4, a toolkit for the simulation of the passage of particles through matter, was developed by the Geant 4 collaboration as a successor to Geant3. The application includes high energy physics, nuclear and accelerator physics. It is also used in medical and space science. GATE (Strul D et al., 2003) is another Monte Carlo software package developed by the international OpenGATE collaboration; it is dedicated to the numerical simulations in medical imaging, and it currently supports PET, SPECT and Computed Tomography (CT). GATE is designed as an user-friendly script of Geant4. Thus, interactions of particles with matters used in GATE are the equivalent of those in Geant4. The relation between GATE and Geant4 is similar to the relation between shell and kernel in Unix/Linux system. The use of GATE is authorized and widespread in PET/SPECT study; however, in this study we worked with Geant4 directly. 


\subsection{PET/SPECT}

PET/SPECT helps elucidate pathologic conditions, and is used in both clinical and research contexts. The applications include oncology, neurology, cardiology and psychiatry. Both PET and SPECT modalities can take images of the distribution of pharmaceuticals labeled with radioisotopes by detecting emitted gamma rays. Thus, in contrast to CT and Magnetic Resonance Imaging (MRI), PET/SPECT provides not morphological images but rather functional images of the pharmaceuticals. Because of their shorter half-lives, it is more difficult to use PET isotopes [F-18 (110 min), O-15 (122 sec), C-11 (20 min), and N-13 (10 min)] than SPECT isotopes [Tl-201 (3 day), I-123 (13 h), and Tc99m (6 h)]. Due to their shorter half-lives, a facility that employs PET must have a cyclotron to produce PET isotopes. This requirement prevents a more rapid increase in clinical use of PET. Meanwhile, SPECT isotopes can be delivered by radiopharmaceutical supply companies. However, PET has better spatial resolution than SPECT as well as better sensitivity. In addition, PET is excellent for quantification. With PET, it is possible to obtain quantitative images with high accuracy, which is important for enhanced sensitivity and specificity in diagnosis. Quantitative images consist of a distribution of absolute values of cerebral and myocardial blood flow; Standard Uptake Value (SUV), an index used in cancer diagnosis; receptor occupancy rate; etc. Techniques of quantification have been under development, and the Monte Carlo method also has played an important role in the development.

The processes to create images in PET/SPECT are described briefly as follows, and shown in Fig. 1. In a PET system, detectors are arranged surrounding a subject. The radiopharmaceutical radiates a positron, and two annihilation gamma rays are emitted with back-to-back angular distribution. The detectors catch the two gamma rays simultaneously (coincidence counting). The line joining two detection points is called the Line of Response (LOR); the LOR tells us the isotope exists somewhere along the line. In a SPECT system, a gamma-ray detector with a collimator made of lead moves around a subject. The LOR is determined by the incoming direction. Next, projection images are created every few degrees. In a SPECT system, we need projection images ranging from 0-360 degrees. In contrast, a PET system requires projections ranging from $0-180$ degrees. A two-dimensional map of projection data against degrees is called "sinogram". Finally, image reconstruction algorithm converts the sinogram into an image of pharmaceuticals distribution. Filtered Back Projection (FBP) and Ordered Subset Expectation Maximization (OSEM) are among the basic methods used in PET/SPECT image reconstructions.

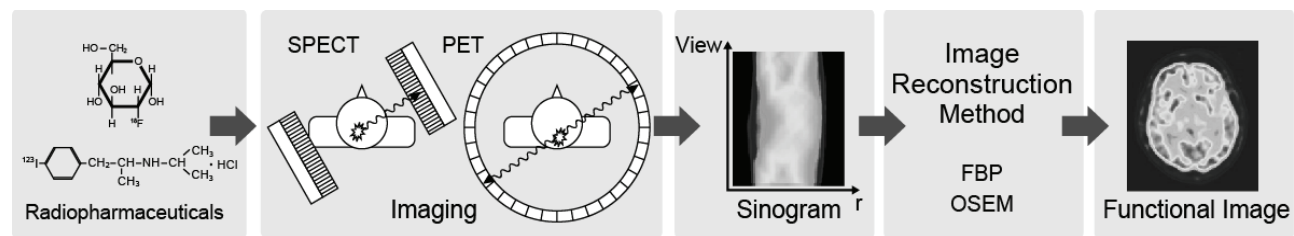

Fig. 1. The procedures for creating functional images in PET/SPECT. Dosed radiopharmaceuticals (tracer) emit annihilation gamma rays, and these are coincidentally detected by scintillators arranged in a circular pattern (PET). In SPECT, tracers radiate single gamma rays, which are collimated and detected by rotating detectors. A set of projections at several degrees, called sinogram, is used for image reconstruction. 


\section{Application to simulation of scintillation lights in SPECT}

\subsection{Introduction}

A SPECT system mainly consists of gamma-ray detectors, the rotating mechanisms and a data acquisition system. Gamma-ray detectors for clinical SPECT are based on scintillators, a number of photo multiplier tubes (PMT) and a collimator. The scintillators are conventional detectors of nuclear radiation; when gamma rays or other particles interact with scintillators, optical photons are generated at the point of interaction. A particle is detected by sensing the scintillation photons. The interaction points are identified by the distribution of scintillation photons on the PMTs. PMTs linearly convert photons into electric charge. The amount of obtained scintillation photons corresponds to the energy deposited by particles in the scintillator. The mean position of PMTs weighted by the number of detected photons is assumed to be the location of interacting points; this frequently used method is called the Anger method. In an actual system, electric resistances are attached to each PMT; the total resistance at both ends is used as the weighting factor. The intrinsic spatial resolution of the Anger method depends on the number of observed scintillation photons and their distribution on the PMTs. The distinct distribution results in better spatial resolution. In order to estimate spatial resolution in SPECT, we should simulate the action of optical photons in a scintillator. We employed Monte Carlo simulation to simulate scintillation photons, and estimated the spatial resolution. Once the simulation is validated, it will be a strong and reliable tool to estimate the performance of a detector. First, in order to determine the adequacy of the simulation, we compared spatial and energy resolutions derived from the simulation with those of an experiment obtained by a high-resolution SPECT, which we are currently developing. In addition, in order to improve spatial resolution, we proposed an alternative to the Anger method. The details of the method and the performance estimation by the simulation are described.

\subsection{High-resolution SPECT for the human brain}

We have been developing a high-resolution and quantitative SPECT for human brain, the concept described in (Zeniya T et al., 2009). The system includes two types of detectors. The first of these (large FOV detector) views the whole human brain with a parallel collimator, and the second (small FOV detector) views a specified local region with extremely high resolution $(\sim 1 \mathrm{~mm})$. To achieve high resolution in SPECT, adoption of a pin-hole collimator is a common method. However, when the FOV (field of view) is smaller than the subject, as during imaging using a pin-hole collimator, quantification is not assured and is often overestimated. The main problem is due to truncations. To compensate for the overestimation, a reconstruction method has developed (Zeniya $\mathrm{T}$ et al., 2007) based on a theory proposed by (Kudo $\mathrm{H}$ et al., 2008). In this method, a reconstructed image without truncations, obtained by the large FOV detector, is used as an initial image for reconstruction by the small FOV detector. We already have completed the large FOV detector; the small FOV detector is still under development. The large FOV detector consists of a $\mathrm{NaI}(\mathrm{Tl})$ scintillator $(147 \mathrm{~mm} l \times 252 \mathrm{~mm} h \times 6.4 \mathrm{~mm} w)$ and 15 flat panel-type multianode PMTs (H8500 Hamamatsu). An H8500 has $8 \times 8$ anodes with $5.8 \mathrm{~mm}^{2}$. The 15 PMTs are arranged in a $5 \times 3$ matrix. In other words, photo-detectors with $5.8 \mathrm{~mm}^{2}$ are located in a $40 \times 24$ matrix. Fifteen PMTs are coupled with the scintillator using optical grease (BC630). The scintillator is covered with a white diffuse reflector to prevent leakage of scintillation photons. The connection side of the PMTs is attached using an optical 
window. $\mathrm{NaI}(\mathrm{Tl})$ must be housed because the material is deliquescent. An electric resistance array used for the Anger method is connected with each anode. The energy spectrum is computed from the total charge of PMTs. The interaction points are calculated using the output of all anodes.

\subsection{Experimental performance evaluation}

\subsubsection{Experimental set up}

To measure spatial and energy resolutions and their position dependence within the detector, we designed a special collimator. The collimator has 170 holes of $1.5 \mathrm{~mm}$ diameter, and holes arranged in a $17 \times 10$ grid pattern at $15 \mathrm{~mm}$ intervals. The collimator is made of lead of $10 \mathrm{~mm}$ thickness, and is mounted on the scintillator. Above the each hole, point sources of Tc- $99 \mathrm{~m}$, which is frequently used in clinical SPECT are placed. Tc-99m mainly emits a single gamma-ray of $140 \mathrm{keV}$.

\subsubsection{Analysis}

The analytical methods used to calculate spatial and energy resolutions are as follows: (i) Select the events from the $140 \mathrm{keV}$ photopeak. The events in a 3-sigma region, as determined by fitting a Gauss function, are extracted. (ii) Using the selected events, interaction points are calculated by the Anger method. (iii) The planar image is projected in the $x$ and $y$ directions. (iv) The spatial resolutions in the $x$ and $y$ directions are derived by fitting a function expressed as follows:

$$
f(x)=\int g(x) \cdot h(z-x) d x=\frac{1}{2 a}\left\{\operatorname{erf}\left(\frac{a+b-x}{\sqrt{2} \sigma}\right)-\operatorname{erf}\left(\frac{b-x}{\sqrt{2} \sigma}\right)\right\},
$$

$f(x)$ is a convolution of a uniform function and a Gauss function. The uniform function represents a collimator hole, and the Gauss function expresses blurring. $a$ is the diameter of a hole, and $b$ is the position of a hole edge. The spatial resolution, Full Width Half Maximum (FWHM), expressed in $\mathrm{mm}$, is computed as $2.35 \times \sigma$, where $\sigma$ is the standard deviation of the Gauss function. (v) Finally, energy resolution at each hole is calculated. The events within a 3-sigma range in position, determined in step (iv), are used to generate the energy spectrum. These energy resolutions (FWHM \%) are derived from fitting of a Gauss function.

\subsubsection{Results}

The planar image of 170 point sources is shown in Fig. 2. Around the center of the detector, equal intervals were obtained, but at the edge of the detector, intervals between neighbors shrink, resulting in worse spatial resolution. The mean spatial resolutions in the $x$ and $y$ direction were, respectively, $3.5 \pm 0.3 \mathrm{~mm}$ and $3.1 \pm 0.3 \mathrm{~mm}$; resolutions at the edge were not used for calculation of the mean values. The best resolutions in the $x$ and $y$ direction were $3.0 \mathrm{~mm}$ and $2.7 \mathrm{~mm}$, respectively. These values were obtained at the center. Meanwhile, mean energy resolution was $10.3 \pm 0.2 \%$, and the best value was $9.9 \%$. We did not observe a distinct position dependence of the energy resolution.

\subsection{Simulation of scintillation lights}

\subsubsection{Geometrical configuration of the simulation}

We used Geant4 (version 9.2) to simulate scintillation photons, and estimated the spatial and energy resolutions of the large FOV detector. Geant 4 takes into account not only 

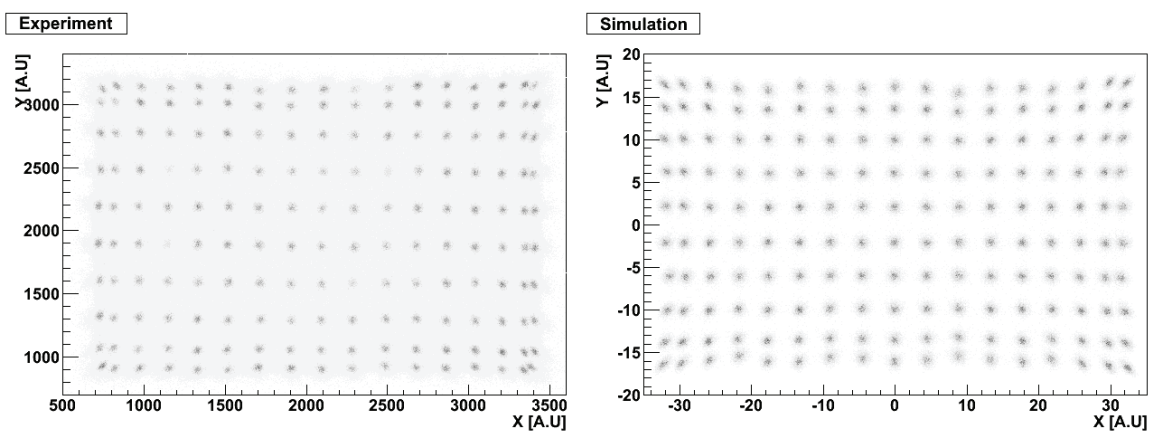

Fig. 2. Planar images of the experiment (left) and the simulation (right). Both results show equal intervals around the center, but shrinkage at the edges.

electromagnetic interactions but also optical photon processes. In this simulation, electromagnetic processes include Compton scattering, Rayleigh scattering, photo-electric effect, ionization, and bremsstrahlung. The first three processes involve gamma rays, and the rest involve electrons. Optical photon processes include absorption, Rayleigh scattering, and boundary processes (refraction/reflection). The details of the optical photon processes are described in next section. Simulation of scintillation photons is difficult; it takes long time due to the large number of optical photons in an event. If a gamma ray interacts with a $\mathrm{NaI}(\mathrm{Tl})$ scintillator, 38000 scintillation photons/ $\mathrm{MeV}$ are generated, all of which must be traced. Moreover, there are many uncertainties (e.g., optical properties of materials). The quantum efficiency of the photo-detector, emission spectrum of the scintillator, and the amount of scintillation photons per unit of energy all must be specified. Strictly speaking, these data have a wavelength dependence. Geant 4 does not include these optical properties; hence, in order to conduct an accurate simulation, we must provide them. When estimating the spatial and energy resolutions, it is critical to obtain the distribution of the scintillation lights on the PMTs. We assembled optical property data from brochures and vendor site as much as possible, and fed these values to Geant4.

The simulation processes are as follows: (i) The geometrical configuration shown in Fig. 3 depicts a $\mathrm{NaI}(\mathrm{Tl})$ scintillator and 15 PMTs (H8500). Optical window, optical grease, and PMT window are also included between the scintillator and the PMTs. Both the optical and PMT windows are made of borosilicate glass, of thickness $3 \mathrm{~mm}$ and $1.5 \mathrm{~mm}$, respectively. The thickness of the optical grease was set as $1 \mathrm{~mm}$. (i) A gamma-ray beam enters the scintillator vertically from above the 170 holes. The source distribution is circular, with a diameter of $1.5 \mathrm{~mm}$. (ii) The gamma ray interacts with the scintillator and deposits its energy. (iii) Scintillation photons are emitted isotropically. The quantity of scintillation photons corresponds to the energy deposited. (iv) The scintillation photons propagate in the scintillator and other materials until they reach the anodes or are absorbed. (v) Some of the scintillation photons reach an anode. The detection of the photon is calculated according to the quantum efficiency and collection efficiency of the H8500. The anodes are assumed to be a perfect absorber. (vi) The interaction point is calculated using the photon distribution on the anodes. Fluctuations of electrical resistance values and anode gains are considered in this simulation. The resistance error and anode gain fluctuation are assigned values of $1 \%$ and $17 \%$, respectively, of the number of observed photons. The anode gain fluctuation is derived from the uniformity map of the H8500 (HAMAMATSU, 2007). The given transmittances of $\mathrm{NaI}(\mathrm{Tl})$ and borosilicate glass are shown in Fig. 4 (right). A dead space, 
which surrounds the anodes, is assumed as a perfect absorber. The refractive indexes of $\mathrm{NaI}(\mathrm{Tl})$, borosilicate glass, and optical grease are 1.85, in Fig. 4 (right), and 1.45 (SaintGobain, 2005-10), respectively. The emission spectrum of $\mathrm{NaI}(\mathrm{Tl})$, together with the quantum efficiency, are shown in Fig. 4 (left). The collection efficiency of the H8500 is assigned a value of $60 \%$. In the case of $\mathrm{NaI}(\mathrm{Tl}), 38000$ photons/ $\mathrm{MeV}$ are produced in each interaction. The reflectivity of the white diffusive reflector is 0.95 (Saint-Gobain, 2004-8). Gamma rays from 3000 events enter the scintillator at 170 holes. The experimental analysis, mentioned in the previous section, is applied to the result of the simulation. This simulation was performed on a personal computer (Linux operating system installed on a $2.4 \mathrm{GHz}$ Intel Core 2 Quad with 2 GB of memory). The computing time for 3000 events was $\sim 4000 \mathrm{sec}$ without parallel computation.
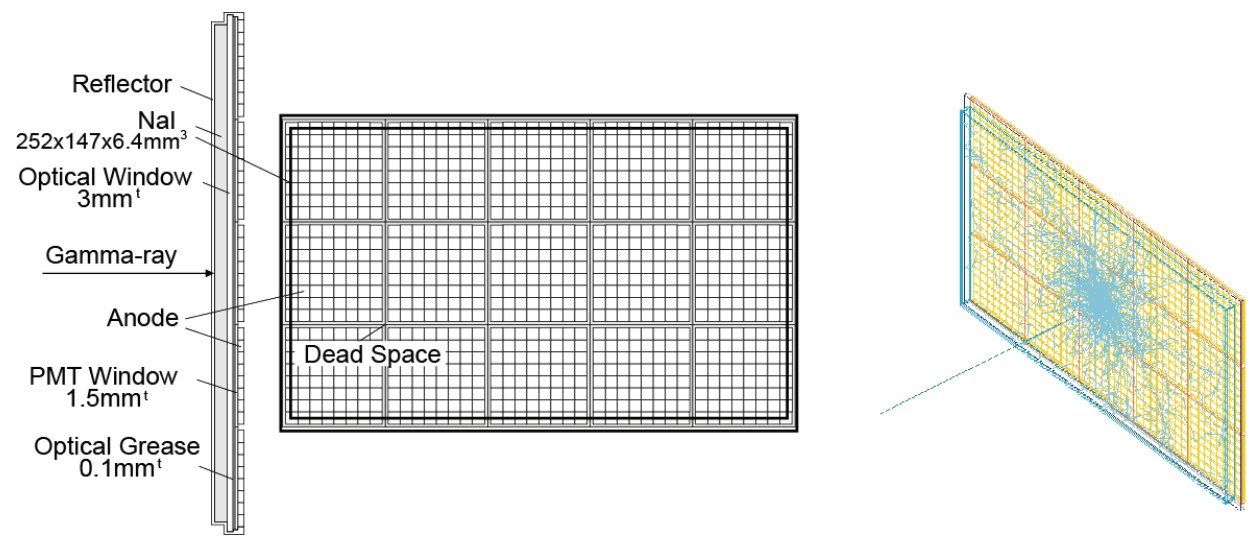

Fig. 3. Simulation geometry of the large FOV detector based on a $\mathrm{NaI}(\mathrm{Tl})$ scintillator and position sensitive PMSs (H8500) (left). The optical window, optical grease, and PMT window are included. The scintillator is covered with white diffusive reflector, except on the side connected to the PMTs. The right figure shows a visualization of the simulation. A gamma ray enters the center of the detector. Scintillation photons are emitted isotropically at interaction points.
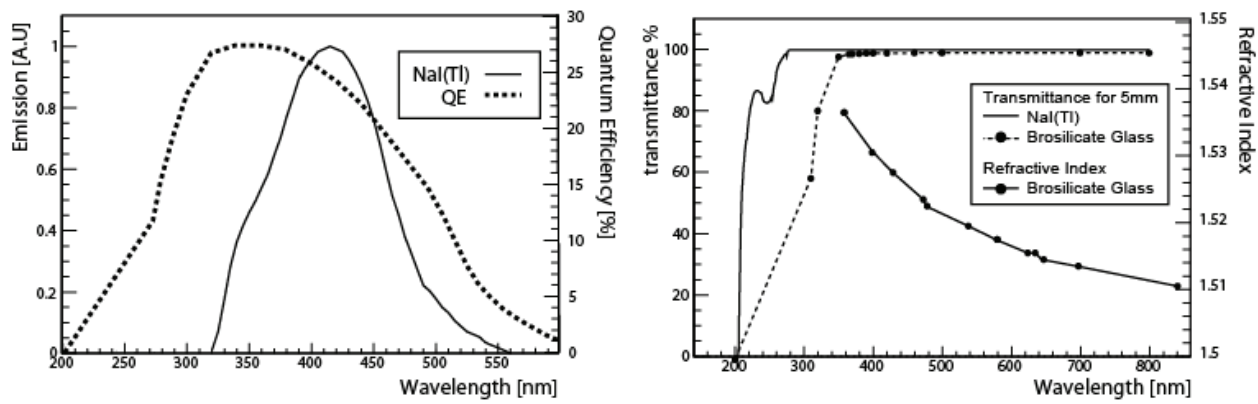

Fig. 4. Optical properties of materials used in this simulation. Emission spectra of $\mathrm{NaI}(\mathrm{Tl})$ scintillator (Saint-Gobain, 2005-8) together with quantum efficiency of H8500

(HAMAMATSU, 2007) (left). Transmittance of NaI(Tl) scintillator and borosilicate glass, and its refractive index (ISP OPTICS) (right). 


\subsubsection{Optical photon process in Geant4}

Geant4 can handle optical processes that include G4OpAbsorption, G4OpBoundaryProcess, G4OpRayleigh, and G4OpWLS (wavelength shift). Absorption, Rayleigh scattering and wavelength shift (if registered) are competing processes in the propagation of optical photons. The absorption depends on absorption length, which must be input by the user. If not given, the material is assumed to be perfectly transparent. The cross-section of the Rayleigh scatter also must be input; however, if the material name is specified as "Water", the cross-section is calculated automatically. To include a wavelength shift process, absorption length and emission spectrum of a wave length shifter are required. A time profile of the emission can be input by the users. At a boundary between different materials, G4OpBoundaryProcess is called. Geant4 has two surface types: dielectric_metal (dielectric-metal interface, in which reflection or absorption is applied), and dielectric_dielectric (dielectric-dielectric interface, in which reflection or refraction or absorption is applied); three types of surface model: GLISUR (original Geant3 model), unified (Levin C. M et al., 1996), and LUT (look-up table model available in version 9.3 and more); and six types of surface finish: polished (smooth perfectly polished surface),

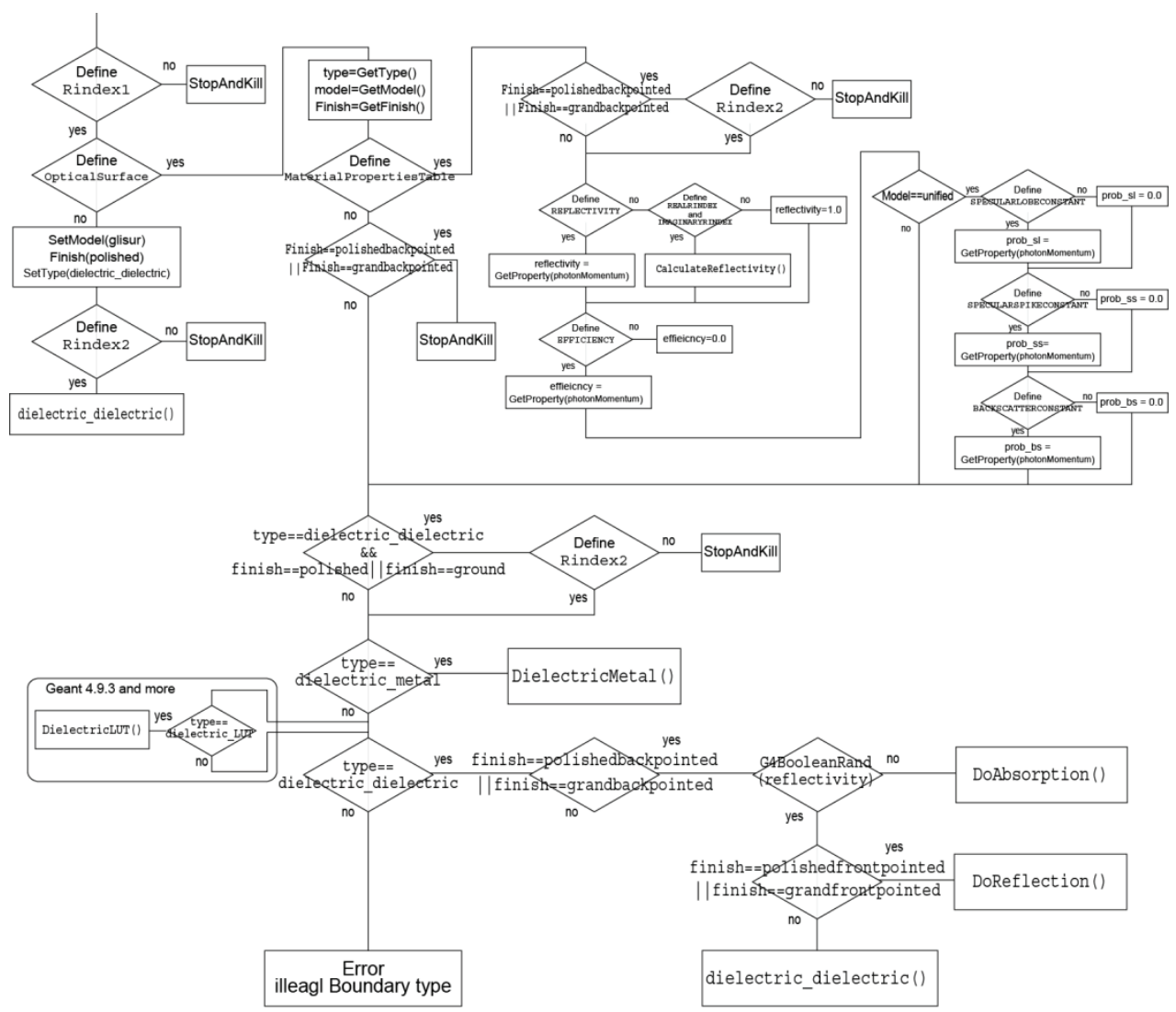

Fig. 5. Flow chart to decide the action of a photon at a boundary. In Geant4.9.3, type of dielectric_LUT is available. In the type, measured angular distributions are used. 
polishedbackpainted (smooth top-layer front paint), polishedbackpainted (same as polished but with a back-point), ground (rough surface), grandfrontpainted (rough top-layer front point), and groundbackpainted (same as ground but with a back-point). The unified model, which considers microfacets of the surface, contains four types of reflections: Lambertian reflection, lobe reflection, spike reflection, and back-scattering. The degree of angular fluctuation is parameterized. Depending on these parameters, action at a boundary is determined as refraction, reflection or absorption. Relation between surface parameters and photon behavior at a boundary is somewhat complex. A flow chart illustrating decisions about this relationship is shown in Fig. 5. In this simulation, the surface parameters of the white diffuse reflector are set as follows: unified model, backfrontpointed, and delectric-dicelectric. The parameter of the rough surface is set as 1.0. In this case, a photon is reflected as diffuse reflection.

\subsubsection{Results}

The planar image of the simulation is shown in Fig. 2, together with the experimental results. The simulation showed the same tendency as in the experiment (equal interval at the center, shrinkage at the edge). The mean spatial resolutions (FWHM) in the $x$ and $y$ directions were $3.3 \pm 0.2 \mathrm{~mm}$ and $3.0 \pm 0.2 \mathrm{~mm}$, respectively, The best resolutions were 2.8 $\mathrm{mm}$ and $2.6 \mathrm{~mm}$, respectively, in the $x$ and $y$ directions. The mean energy resolution (FHWM) was $9.1 \%$, and the best resolution was $8.5 \%$. An energy spectrum of the center is shown in Fig. 6 alongside the spectrum obtained by experiment. The comparisons of the resolutions are listed in Table 1.

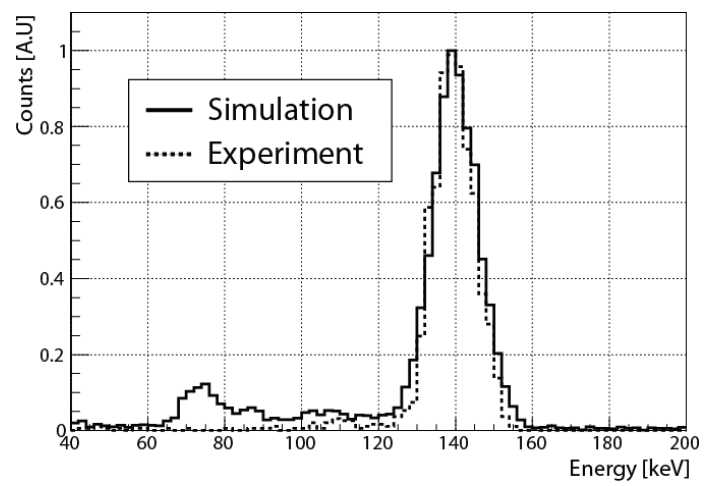

Fig. 6. Energy spectra from experiment and simulation, obtained at 5 th row and 6th column. Experimental and simulation resolutions are $10.1 \%$ and $9.2 \%$, respectively.

\begin{tabular}{lrr}
\hline Resolution (FWHM) & $\begin{array}{c}\text { Experiment } \\
\text { Mean } \pm \text { S.D (Best) }\end{array}$ & $\begin{array}{c}\text { Simulation } \\
\text { Mean } \pm \text { S.D (Best) }\end{array}$ \\
\hline Spatial (X-dir) [mm] & $3.5 \pm 0.3(3.0)$ & $3.3 \pm 0.2(2.8)$ \\
Spatial (Y-dir) [mm] & $3.1 \pm 0.3(2.7)$ & $3.0 \pm 0.2(2.6)$ \\
Energy [\%] & $10.3 \pm 0.2(9.9)$ & $9.1 \pm 0.3(8.5)$ \\
\hline
\end{tabular}

Table 1. Comparison of spatial and energy resolutions between simulation and experiment. The mean values are calculated without edge resolutions. The parenthetical values are the best resolutions, obtained at the center. 


\subsubsection{Discussion}

The spatial resolutions of the simulation are consistent with those of the experiment (within $10 \%)$. The accuracy seems to be sufficient to estimate resolutions and design new detectors. The simulation also has good agreement with experiment with regard to energy resolution. The energy spectrum is formed from the quantity of obtained photons. The energy resolution of simulations is usually generated using an adjustment parameter decided by an experiment. In this work, however, the energy resolution is calculated without such a parameter, depending on the number of photons. The difference between spatial resolutions in the $x$ and $y$ directions was obtained in both results. We obtained $3.5 \mathrm{~mm}$ and $3.1 \mathrm{~mm}$ in $x$ and $y$-directions, respectively, in the experiment. This difference is due to the difference in the number of anodes. Propagation of fluctuation of anode output in the $x$ direction is larger than that of the propagation in the $y$ direction. If a detector has the same number of anode in the $x$ and $y$ directions, the spatial resolutions are comparable, as shown by another simulation (not described in this chapter). Available clinical SPECTs commonly have $\sim 4 \mathrm{~mm}$ spatial resolution (FWHM) with a smaller number of PMTs (or anodes). It seems using many anodes does not make much contribution to the improvement of spatial resolution. The large error propagation of anode output deteriorates resolutions.

A simulation is useful for the design of a detector. It is easy to change the thickness or type of scintillator, photo-detector, or other parameters in the simulation. Moreover, the simulation is able to identify the main absorber and the main causes of deterioration of resolutions. As the demonstration, we replaced optical grease with air, whose refractive index is $\sim 1.0$. The variations in the absorption fraction of materials relative to total generated photons and number of total internal reflections are listed in Table 2. Because of the disjunction of refractive index at a boundary, the number of total internal reflection increases. The increase in total reflections creates a longer path length for photons in the scintillator, resulting in absorption by the scintillator. About $80 \%$ of total generated photons reached at PMT anodes, and $20 \%$ of reached photons were obtained.

\begin{tabular}{lrr}
\hline & $\begin{array}{c}\text { Boundary of } \\
\text { optical grease }\end{array}$ & $\begin{array}{l}\text { Boundary of } \\
\text { Air }\end{array}$ \\
\hline Detection & $12 \%$ & $10 \%$ \\
PMT Anode & $73 \%$ & $62 \%$ \\
PMT Window & $0.20 \%$ & $0.12 \%$ \\
PMT Dead Space & $5.6 \%$ & $6.3 \%$ \\
Reflector & $6.9 \%$ & $16 \%$ \\
NaI scintillator & $0.91 \%$ & $1.84 \%$ \\
Optical Window & $0.73 \%$ & $1.2 \%$ \\
Optical Grease & $\sim 0 \%$ & $\sim 0 \%$ \\
Total internal reflection & 1 & 14.7 \\
\hline
\end{tabular}

Table 2. Absorption fraction of materials (\%). Detection means a photon reaches at an anode and obtained according to quantum efficiency and collection efficiency. If not obtain, a photon absorbed by a anode (PMT Anode). Number of total internal reflections is normalized against that of optical grease.

The simulation results are always overestimated. Our simulation still does not consider other uncertainties, e.g., fluctuation of the number of electrons in the multiplying process of a PMT, etc. In addition, there are unknown optical properties such as the absorption length 
of the optical grease (in this study, however, the absorption can be treated as negligible because the grease layer is thin) and almost the properties does not have wavelength dependence. We need a dataset of optical properties of materials, especially those used in scintillation detectors. In order to conduct accurate simulations, it seems better to consider other uncertainties as much as possible. Although some optical properties are neglected, this simulation already has sufficient reproducibility.

\subsection{Simulation approach to improve spatial resolution}

\subsubsection{Methods}

We aim at spatial resolution of $\sim 2 \mathrm{~mm}$ in the large FOV detector to make a difference from clinical SPECTs. However, we obtained a resolution of $\sim 3.5 \mathrm{~mm}$, which is worse than expected. Therefore, we proposed an alternative to the Anger method to improve the spatial resolution. The method is simple. Previously, photon distributions on anodes were measured at every known position in the detector. In this work, we calculated the distribution of gamma rays on a grid pattern with $1 \mathrm{~mm}$ intervals. Each distribution is obtained from average of incident 300 gamma rays. The number of total distributions is $37296(252 \times 148)$. One quarter was simulated, and the rest were derived from symmetrical properties. The set of distributions is used as reference data. Every time scintillation photons are radiated, the distribution is compared with the reference dataset. The position of most similar distribution, determined by the least square algorithm, is identified as the point of interaction. In an actual experiment, a reference dataset can be accumulated by scanning collimated gamma rays. We evaluated the usefulness of the method. The result of the simulation in the validation study is reusable for this evaluation.

\subsubsection{Results}

Examples of reference data are shown in Fig. 7. Fig. 7 shows photon distributions in the case that gamma rays enter at the center (left) or at the edge (right). The planar image and projection in the $y$ direction are shown in Fig. 8. In contrast to the Anger method, constant intervals were obtained among grid points. At the edge, however, a point divided into two.
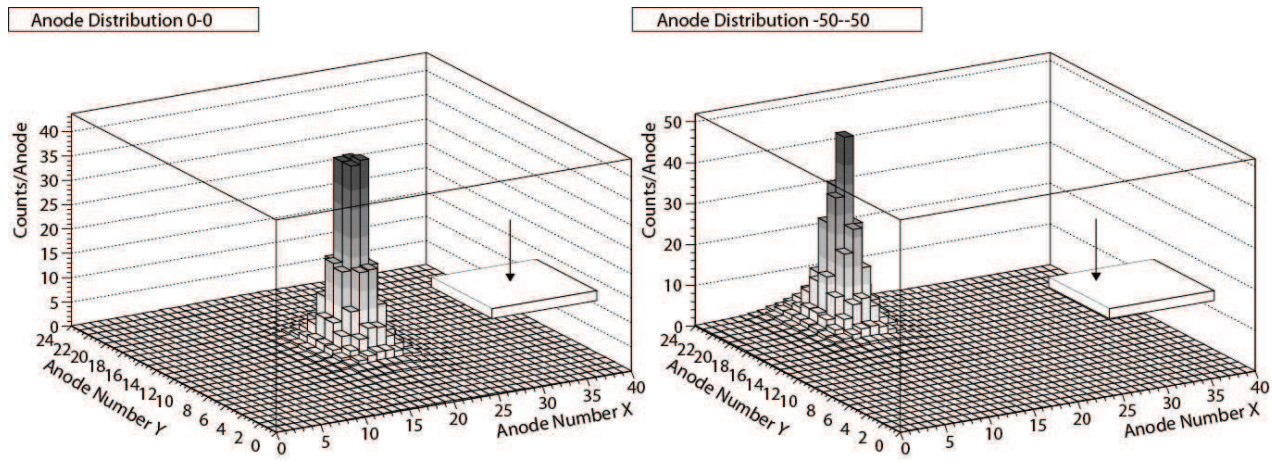

Fig. 7. Examples of photon distribution on the anodes of 15 H8500s. The left figure shows the distribution in the case that gamma rays enter from the center; the right figure shows the case in which the gamma rays enter from $(-50 \mathrm{~mm},-50 \mathrm{~mm})$ relative to the center. We calculated photon distributions, which are obtained from the known positions of gammaray sources. The set of distributions was used as reference data to decide interaction points. 
The mean spatial resolutions, without the edge resolutions, were $1.8 \pm 0.8 \mathrm{~mm}$ and $1.8 \pm 0.8$ $\mathrm{mm}$ in the $x$ and $y$ directions, respectively. The mean energy resolution is $10 \pm 5 \%$. An obvious position dependence of the energy resolution was not obtained using this method.
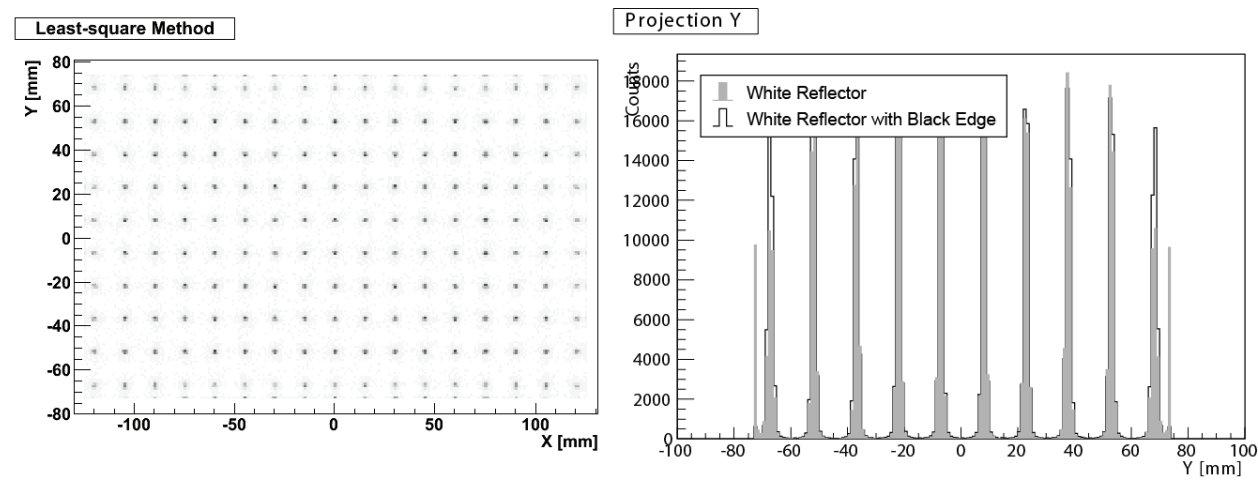

Fig. 8. Planar image obtained by the proposed method using the least-square algorithm. (left), and the projections to the $y$ direction (right). The gray filled histogram is the projection with a white diffusive reflector; the white filled histogram is a projection with a black edge reflector. Misidentifications, especially at the edge, were improved by using the black edge white reflector.

\subsubsection{Discussion}

The proposed method indicated improvement of spatial resolution from $3.5 \mathrm{~mm}$ to $1.8 \mathrm{~mm}$ in the $x$ direction. The large FOV detector has the potential for high resolution, $\sim 2 \mathrm{~mm}$. One advantage of this method is that the unit of the planar image is actual length (mm); this is different from the Anger method, in which the unit is arbitrary. We do not need to calibrate position.

At the edge, many misidentifications are seen due to small difference of reference dataset at the edge. To prevent misidentification, it is useful to employ a diffuse reflector with black edge (the side of the scintillator is coated with absorbent material). The projection with the black edge reflector is shown in Fig. 8, together with the original reflector. The false identifications were corrected at the edge. Meanwhile, the energy resolutions at the edge became worse due to a reduction in the number of photons by absorption.

To implement this method, it is essential to install an analog to digital converter (ADC) for each anode. This conversion may take a great deal of time and decrease the costeffectiveness. However, it is worthwhile to try this method, because once all anode outputs are converted, we can try other methods. A neural network would be one promising method; another would be to employ a modified Anger method using restricted anodes that are peripheral to the largest output anode. We are developing a full digital system for the method. Furthermore, if the reference data is compiled using only simulations with high accuracy, the technique has the potential to discriminate the depth of interactions. It sometimes happens that a gamma ray is scattered before absorbed in the scintillator. In this case, there are multiple sources of scintillation photons, and these events impair spatial resolution. It is difficult to discriminate events with multiple scintillation sources from 
events with single scintillation source. Chi-square, defined by $\chi^{2}=\frac{1}{n d f} \sum_{i} \frac{\left(o b j_{i}-r e f_{i}\right)^{2}}{\text { error }_{i}^{2}}$, may help to make the distinction; here, $i, o b j$, ref, error $n d f$ mean index of anode, anode output, anode output of reference data, error of anode output, and number of degrees of freedom, respectively. A large chi-square indicates an event with multiple scintillation sources. Higher-energy gamma rays, such as $511 \mathrm{keV}$, tend to be such a event. In a SPECT system, however, because of the low energy of gamma-rays (in the range of several hundred $\mathrm{keV}$ ), these occurrences are rare, and can safely be ignored.

\subsection{Conclusion}

Our simulation is in good agreement with the experiment that determined the intrinsic spatial and energy resolutions of the large FOV detector. The proposed method, using ADCs for all anodes, is promising. We expect that this will improve spatial resolution from $\sim 3.5$ $\mathrm{mm}$ to $\sim 1.8 \mathrm{~mm}$; it also has prospects for use in other identification methods.

The simulation including action of scintillation lights will be helpful for a design of a new SPECT by estimation of the spatial resolution. However, we still used insufficient data regarding optical properties in this work. We hope that data regarding the properties of scintillators and materials are in public.

\section{Application to a quantification technique in PET}

\subsection{Introduction}

Quantification in PET is important for improvement in diagnosis. Here, quantification refers to the absolute pixel value in PET images. Examples of quantitative values in PET include SUV, cerebral and myocardial blood flow, cerebral metabolic rate of oxygen, oxygen extraction fraction in ischemia check. An accurate quantification can prevent erroneous diagnosis and provide high-quality data for multi-center studies. However, there are some factors that hamper quantification; these include random events (accidental events) and scatter events. Random events are accidental coincidental counts that originate from two different sources. The scatter events also reflect coincidence counting, but in these cases one or both gamma rays have been scattered. Gamma rays lose their energy by Compton scattering, and it is easy to discriminate the events by selecting the $511 \mathrm{keV}$ photopeak. However, because of the poor energy resolution of detectors, it is impossible to remove all scatter events. These events result in inaccurate LORs and disturb both image quality and quantification. The random events can be corrected by a delayed coincidence technique. The scatter events are also compensated by some kinds of correction theories. However, when significant activity exists outside the FOV (typical PETs have 15-25 cm FOV), verification of scatter corrections in 3D acquisition mode has not yet been thoroughly investigated. Measurements of myocardium blood flow represent an illustrative example. The liver, located outside the FOV, has larger activity than that of the heart, which is within the FOV. The uncorrected scatter events originating from outside the FOV may reduce the accuracy of quantification. One of the advantages of simulations is that they allow an understanding of processes that are difficult or impossible to know in experiments. Scatter events are also difficult to identify in experiments. We conducted a simulation (Geant4 version 9.2) of scatters with a numerical human model, and investigated the effects of scatter events on quantification in cardiac PET using O-15 water. 


\subsection{PET configuration in the simulation}

We reproduced a realistic configuration of a PET (ECAT ACCEL, Siemens) (Herzog H et al., 2004), which is installed in our facility. The PET is based on LSO scintillators consisting of a $2.5 \times 2.5 \times 6 \mathrm{~mm}^{3}$ rectangular solid. The detector consists of 9216 scintillators, which are circularly arranged. One ring consists of 384 scintillators, and 24 rings can be arranged in the axial direction. The diameter of the ring is $824 \mathrm{~mm}$, and the aperture plane of the gantry is $562 \mathrm{~mm}$. The FOV is $162 \mathrm{~mm}$. To simplify, in this simulation, the detector is shaped as a ring with the following dimensions: $824 \mathrm{~mm}$ diameter, $162 \mathrm{~mm}$ width, and $6.5 \mathrm{~mm}$ thickness. The structures contain not only scintillators but also PMTs, front shield, septa, bed, body of equipment, etc., shown in Fig. 9. These are included, because such structures slightly increase scatter events originating from activity outside the FOV. The adequacy of this simulation has been evaluated by comparison of the scatter fraction. Scatter fraction is the ratio between total coincident events and scatter events, measured by using a special phantom (scatter phantom); and the measurement method is described in the NEMA standard (NEMA NU 2-2007). The experimental scatter fraction was $45.0 \%$, and that of the simulation is $45.9 \%$. The simulation reproduced the experiment with high accuracy.

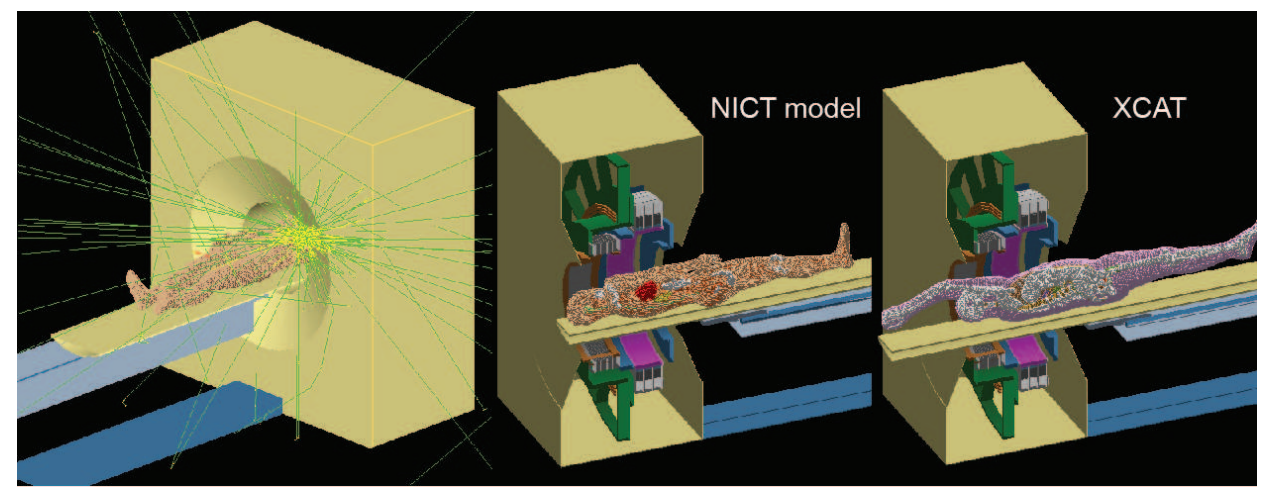

Fig. 9. Geometrical configuration of the simulation in PET. For realistic simulation, we reproduced a PET using the numerical human model generated by NICT (left). The line shows gamma rays. The center and right figures, respectively, show laminagrams of the PET containing the NICT model and XCAT,.

\subsection{Simulation with numerical human model}

For a realistic simulation, we employed a numerical human model (Nagaoka T et al., 2004) developed by National Institute of Information and Communications Technology (NICT) in Japan. The model is based on MRI images of Japanese people with average height and weight: $173 \mathrm{~cm}$ and $65 \mathrm{~kg}$ for male; $160 \mathrm{~cm}$ and $50 \mathrm{~kg}$ for female. The models consist of $320 \times$ $160 \times 866$ voxels for male, or $320 \times 160 \times 804$ voxels for female; voxels are $2 \mathrm{~mm}^{3}$. Fifty-one organs or tissues can be identified, and ID numbers are given to each voxel. In addition, models of a child, a pregnant female and an arbitrary pose have been developed. There is another available numerical human model, the 4D NURBS-based Cardiac-Torso (XCAT*) phantom (Segars WP et al., 2003). The organs are constructed using non-uniform rational bsplines, or NURBS surfaces based on the three-dimensional Visible Human CT dataset. $\mathrm{XCAT}$ is able to calculate cardiac and respiratory motions. It is powerful tool for evaluating 
motion effects, and is widely used in nuclear medicine imaging research. The geometrical configuration with NICT model and XCAT are shown in Fig. 9. To simulate interactions of gamma rays with the human body, we need attenuation coefficients for each tissue. We calculated the attenuation coefficients using compositions and density of the tissues found in a web site of Life Sciences Division at Oak Ridge National Laboratory (ORNL), "Description of the mathematical phantoms" and (Akkurt $\mathrm{H}$ et al., 2007). Compositions and densities of soft tissue, skeleton, lung, adipose, and glandular tissue are available from "Description of the mathematical phantoms"; compositions of bone, muscle, skin, brain, eyes, thyroid, upper face, larynx, trachea, gastrointestinal tract, testes, ovaries, uterus, urinary bladder, spleen, heart, pancreas, liver, kidney, breast, and blood are available from (Akkurt $\mathrm{H}$ et al., 2007). We assigned tissue data listed above to 51 types of tissues. In this work, we used the male NICT model and its 8 voxels are collected up to 1voxel (4 mm cubic) to reduce the computing time.

\subsection{Estimation of scatter in cardiac PET}

In cardiac PET to measure myocardium blood flow, O-15 water, $\mathrm{Rb}-82$, and $\mathrm{N}-13-\mathrm{NH}_{3}$ are used as tracers. O-15 water is one of the most ideal tracers, because it immediately diffuses into tissues and washes out into veins. That behavior makes it easy to calculate myocardium blood flow with a 2-compartment model (Iida H et al., 1992). Activated water is injected and spreads into the whole body, in which the activity of each organ varies with time. At the first phase, the heart has a large activity, and the tracer accumulates in the liver at the late phase. The time activity curve of the heart, liver and lung is shown in Fig. 10, obtained with a clinical examination. The horizontal axis shows time in seconds; the vertical axis shows activity per organ. In PET images, we can obtain a pixel value as activity per volume $(\mathrm{Bq} / \mathrm{cc})$. The activities per organ are derived from experimental data $(\mathrm{Bq} / \mathrm{cc}) \times$ volume of heart (406 cc), liver (3296 cc), and lung (1150 cc), using the NICT model, under the assumption that each organ has uniform activity. In one phase (100 sec in Fig. 10), the liver has 1.5 times the activity of the heart. This phase seems to significantly influence activity outside the FOV. We simulated trajectories of annihilation gamma rays. In this PET study, the scintillation lights are not tracked. Positions of interaction between gamma rays and the scintillator are saved to create a sinogram using the ECAT format for 3D acquisition mode. The image is reconstructed by FBP with the scatter correction algorithm installed in the ECAT image reconstruction system. The image size is a $128 \times 128$ matrix (47 slices). The scatter correction is single scatter simulation (SSS) (Ollinger JM, 1996). To obtain a quantitative image, an attenuation map for the correction of absorptions is required. In this simulation, the attenuation map is calculated analytically. We created three types of image. The first one is an image reconstructed with scatter correction (normal image). The second one is an image using a sinogram without scatter events, which can be discriminated in the simulation (true image). The third one is an image using only scatter events (scatter image). The last two images are reconstructed without the scatter correction. We can obtain only a normal image in an actual PET study. According to the time activity curve, sinograms of the heart, liver and lung are combined in proportion to their activity at each time, and reconstructed into three types of image. Twenty set of images are created. If scatter events are corrected accurately, the normal image should be same as the true image. By means of comparison between the normal and true images, we evaluated adequacy of the scatter correction. Two billion events were performed in each organ. These computations 
took $\sim 420 \mathrm{~h}$ to complete with a personal computer (Linux operating system installed on a 2.4 GHz Intel Core 2 Quad with 2 GB of memory). However, we can reduce the computing time down to $\sim 20 \mathrm{~h}$ using 5 computers with comparable specifications. Twenty processes are able to run in parallel.

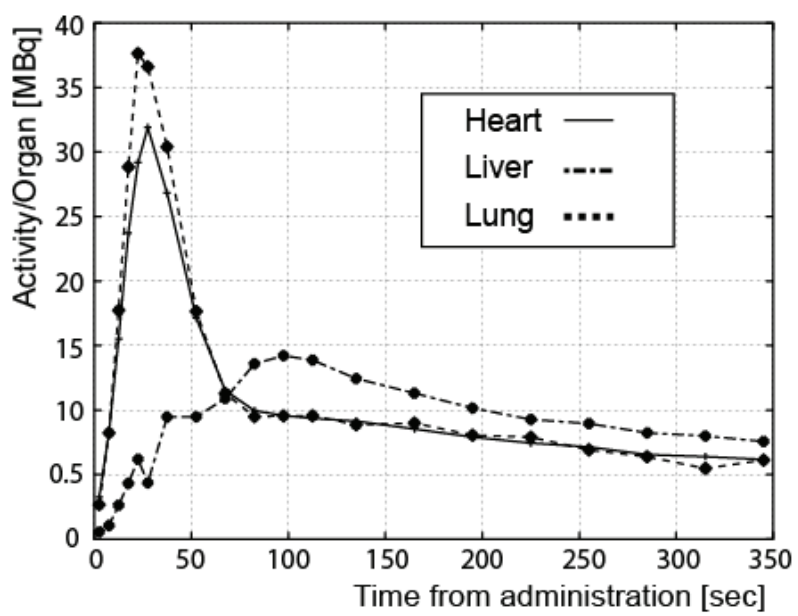

Fig. 10. Time activity curves of the heart, liver and lung. The vertical axis represents the activity of a organ. The horizontal axis represents the time after the administration of O-15 water. For one phase ( 100 sec) the liver (located outside the FOV) has 1.5 times more activity than the heart.

\subsection{Results}

The sets of images of slice 14 (the apex and liver are seen) at 20, 45, 75 120, 210 and 300 sec after the injection are shown in Fig. 12. We placed ROIs (region of interests) on the tissues (anterior, posterior, septal, lateral, and apex) in the normal and true images. The difference between the two ROI values for the apex is shown in Fig. 11. Myocardial blood flows (MBF), which are calculated with time series of ROI values, are listed in Table 3. MBFs of the normal image are normalized by experimental values. The ROI values of normal images overestimate that of the true image due to the insufficient scatter correction. However, myocardium blood flows remained unchanged.

\begin{tabular}{lll}
\hline $\mathrm{MBF}(\mathrm{mL} / \mathrm{min} / \mathrm{g})$ & Normal Image & True Image \\
\hline Apex & 1.09 & 1.11 \\
Anterior & 0.81 & 0.80 \\
Lateral & 1.03 & 1.03 \\
Posterior & 0.80 & 0.79 \\
Septal & 0.75 & 0.73 \\
\hline
\end{tabular}

Table 3. Myocardial blood flow (MBF) calculated using the normal image and true image. The MBFs of normal image are normalized by these of an experimental value. We obtained equivalent vales derived from the normal and true image. 


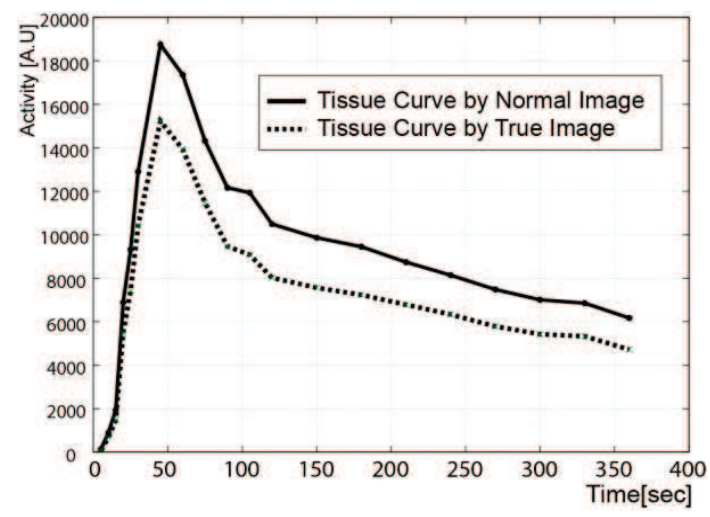

Fig. 11. Time activity curve of a tissue (apex). The solid line is derived from the normal image. The dotted line is derived from the true image. Due to the uncorrected scatters, the normal image is overestimated. At the wash-out phase ( $\sim 50 \mathrm{sec}$ and after) the both curves have almost the same reduction rate.

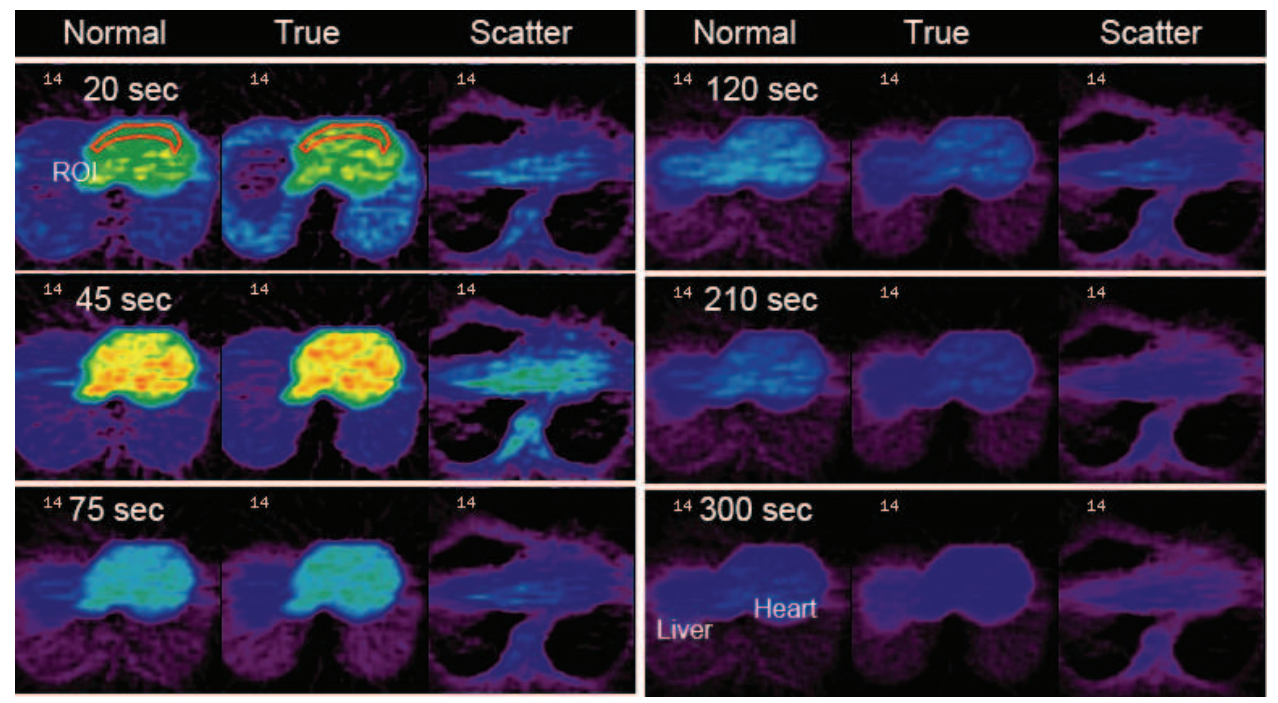

Fig. 12. Reconstructed images of the normal, true, and scatter images at 20, 45, 75, 120, 210, and $300 \mathrm{sec}$ from the injection. At the wash-out phase, scatter distributions are uniform and almost the same in the heart region.

\subsection{Discussion}

In this simulation, large activity outside the FOV influences quantification of an image. The scatters are not compensated accurately; consequently, ROI values tend to overestimate true values. This result indicates that some quantitative physiological parameters measured by PET may be overestimated. However, physiological parameters do not always depend on absolute ROI values. Quantitative myocardial blood flows determined by O-15 seem to epitomize this case. Because the blood flows are calculated by the tissue wash-out rate, 
which is equivalent both in normal images and true images. As shown in Fig. 11, uncorrected scatters make a constant contribution to the ROI values in the heart region at the wash-out phase, and this leaves myocardial blood flow unchanged.

By means of this method, we can evaluate other scatter corrections using other PET configurations. Recently, PET/CT has become a major modality, replacing single PET; in this modality, a CT is installed next to PET detector. The influence of structures of the CT on quantification should be investigated. Our simulation with a realistic geometrical configuration can clarify the influence. In addition, it is useful for estimation of random events. To remove random events originating from activity outside the FOV, it will be essential to improve image quality and reduce measurement dead time. We can design optimized the shielding system by using this simulation.

\subsection{Conclusion}

We simulated scatter events in cardiac PET using an O-15 tracer. In this work, three types of images (normal image, true image, and scatter image) were created using a sinogram obtained with this simulation and the reconstruction method with scatter correction installed in ECAT system. By comparing between the normal image and true image, we obtained that the scatter correction is not sufficient due to the existence of significant activity outside the FOV. However, even in this case, myocardial blood flow (calculated by tracer wash-out rate) stayed unchanged.

\section{Conclusion of this chapter}

In this chapter, applications of Monte Carlo method to PET and SPECT studies were mentioned. In both SPECT and PET studies, the spatial and energy resolutions of SPECT by simulating scintillation photons (and scatter events in PET) reproduced the results of experiments. Monte Carlo simulations can be used as a reliable tool in the development of medical equipments.

\section{Acknowledgment}

The author thanks Professor Hidehiro lida for many helpful discussions, and Dr. Tustomu Zeniya and Dr. Kazuhiro Koshino for their kind assistance. The author also thanks aid Assistant Professor Hiroshi Watabe for his teaching of operation of the PET. The work on the development of a high-resolution SPECT described in early part of this chapter was supported by part of the Newly Adopted Projects of Regional R\&D Programs for FY2008 from the Kansai Bureau of Economy, Trade and Industry, Health Labour Sciences Research Grant; DDS from the Ministry of Health, Labour and Welfare (MHLW); and the Budget for Nuclear Research of the Ministry of Education, Culture, Sports, Science and Technology (MEXT); all funding agencies are in Japan. The work for simulation study in PET was funded by a Grant-in-Aid for Young Scientists (B), also in Japan.

\section{References}

Akkurt H, \& Echerman FK. (2007). Development of PIMAL: Mathematical Phantom With Moving Arms and Legs. ORNL/TM-2007/14

Allison J. et al,. (2006). Geant4 developments and applications, IEEE Transactions on NuclearScience, Vol.53, No1, pp.270-278, 0018-9499 
HAMAMATSU. (2007). H8500 SERESE, October 2010 http://jp.hamamatsu.com/ resources/ products/etd/pdf/H8500C_H8500D_TPMH1308E01.pdf

Herzog H, Tellmann L, Hocke C, Pietrzyk U, Casey ME, \& Kuwrt T. (2004). NEMA NU22001 guided performance evaluation of four Siemens ECAT PET scanners. IEEE Transactions on Nuclear Science, Vol.51, No.5, pp.2662-2669, 0018-9499

Iida H, Christopher GR, Ranil de S, Luis IA, Peter MB, Adriaan AL, \& Terry J. (1992). Use of the left ventricular time-activity curve as a noninvasive input function in dynamic oxygen15-water positron emission tomography. J Nucl Med, Vol.33, No.9, pp1669-1677

ISP OPTICS. BK7 Schott Glass, October 2010 http://www.ispoptics.com/PDFs/PDFCatalog/Page14.pdf

Kudo H, Courdurier M, Noo F, \& Defrise M. (2008). Tiny a prior knowledge solves the interior problem in computed tomography. Phys. Med. Biol., Vol. 53, No.9, pp.2207-2231

Levin A, \& Moisan C. (1996). A More Physical Approach to Model the Surface Treatment of Scintillation Counters and its Implementation into DETECT. TRIUMF Preprint TRI, pp.96-64

Nagaoka T, Watanabe S, Sakurai K, Kunieda E, Watanabe S, Taki M, \& Yamanaka Y. (2004). Development of Realistic High-Resolution Whole-Body Voxel Models of Japanese Adult Male and Female of Average Height and Weight, and Application of Models to Radio-Frequency Electromagnetic-Field Dosmietry. Physics in Medicine and Biology, Vol,49, No.49, pp1-15

NEMA NU 2-2007. Performance Measurement of Positron Emission Tomographs National Electrical Manufactures Association

Ollinger JM. (1996). Model-based scatter correction for fully 3D PET. Phys. Med. Biol. Vol. 41, No. 1, pp.153-176

ORNL. Description of the mathematical phantoms, October 2010 http:/ / ordose.ornl.gov/resources/Mird.pdf

Saint-Gobain. (2004-8). Scintillation Products, October 2010 http://www.detectors.saintgobain.com/uploadedFiles/SGdetectors/Documents/Brochures/Arrays-Brochure.pdf

Saint-Gobain. (2005-10). Detector Assembly Materials, October 2010 http://www.detectors.saint-gobain.com/uploadedFiles/SGdetectors/Documents/ Product_Data_Sheets/SGC_Detector_Assembly_Materials_Data_Sheet.pdf

Saint-Gobain. (2005-8). NaI(Tl) Scintillation Material, October 2010 http://www.detectors.saint-gobain.com/uploadedFiles/SGdetectors/Documents/ Product_Data_Sheets/NaI\%28T1\%29-Data-Sheet.pdf

Segars WP, Tsui BMW, Frey EC, \& Fishman EK. (2003). Extension of the 4D NCAT phantom to dynamic x-ray CT simulation. Nuclear Science Symposium Conference Record, 2003 IEEE, pp.3195-3199, 1082-3654

Strul D, Santin G, Lazaro D, Breton V, \& Morel C. GATE (Geant4 Application for Tomographic Emission): a PET/SPECT general-purpose simulation platform. (2003). Nucl. Phys. B (Proc. Suppl.), Vol.125, pp.75-79

Zeniya $T$, Watabe $H$, Inomata $T$, Iida H, Sohlberg A, \& Kudo H. (2007). 3DOSEM reconstruction from truncated data in pinhole SPECT. 2007 IEEE Nuclear Science Symposium Conference Record, Vol. 6, pp.4205-4207, 1082-3654

Zeniya T, Hirano Y, Sakimoto T, Ishida K, Watabe H, Teramoto N, Kudo H, Minato K, Hatazawa J, \& Iida H. (2009). Conceptual Design of High Resolution and a Quantitative SPECT system for imaging a selected small ROI of human brain. 2009 IEEE Nuclear Science Symposium Conference Record, pp. 3484-3486, 1082-3654 


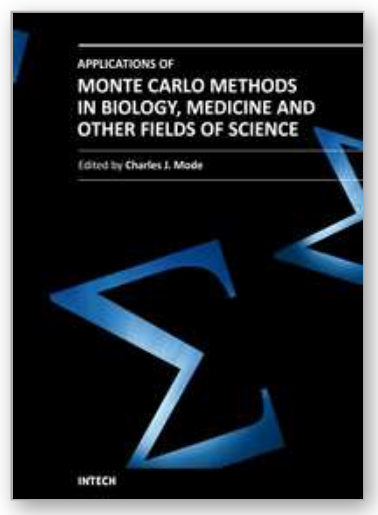

\section{Applications of Monte Carlo Methods in Biology, Medicine and Other Fields of Science}

Edited by Prof. Charles J. Mode

ISBN 978-953-307-427-6

Hard cover, 424 pages

Publisher InTech

Published online 28, February, 2011

Published in print edition February, 2011

This volume is an eclectic mix of applications of Monte Carlo methods in many fields of research should not be surprising, because of the ubiquitous use of these methods in many fields of human endeavor. In an attempt to focus attention on a manageable set of applications, the main thrust of this book is to emphasize applications of Monte Carlo simulation methods in biology and medicine.

\section{How to reference}

In order to correctly reference this scholarly work, feel free to copy and paste the following:

Yoshiyuki Hirano (2011). Applications to Development of PET/SPECT System by Use of Geant4, Applications of Monte Carlo Methods in Biology, Medicine and Other Fields of Science, Prof. Charles J. Mode (Ed.), ISBN: 978-953-307-427-6, InTech, Available from: http://www.intechopen.com/books/applications-of-monte-carlomethods-in-biology-medicine-and-other-fields-of-science/applications-to-development-of-pet-spect-system-byuse-of-geant4

\section{INTECH}

open science | open minds

\author{
InTech Europe \\ University Campus STeP Ri \\ Slavka Krautzeka 83/A \\ 51000 Rijeka, Croatia \\ Phone: +385 (51) 770447 \\ Fax: +385 (51) 686166 \\ www.intechopen.com
}

\author{
InTech China \\ Unit 405, Office Block, Hotel Equatorial Shanghai \\ No.65, Yan An Road (West), Shanghai, 200040, China \\ 中国上海市延安西路65号上海国际贵都大饭店办公楼 405 单元 \\ Phone: +86-21-62489820 \\ Fax: $+86-21-62489821$
}


(C) 2011 The Author(s). Licensee IntechOpen. This chapter is distributed under the terms of the Creative Commons Attribution-NonCommercialShareAlike-3.0 License, which permits use, distribution and reproduction for non-commercial purposes, provided the original is properly cited and derivative works building on this content are distributed under the same license. 